\title{
基于时空临近重复效应的犯罪热点特征及成因分析 以北京市抢劫案件为例
}

\author{
郭雅琦, 陈 鹏* \\ (中国人民公安大学警务信息工程与网络安全学院, 北京 102600)
}

\begin{abstract}
摘 要: 时空临近重复效应是犯罪活动的一种重要时空特征。为深人研究犯罪热点的特征及其形成原因, 论文以北 京市内城六区2012-2014年抢劫案件为例, 通过核密度估计、时空临近重复计算及定义时空临近重复案件链等方 法分析了犯罪热点的案件构成, 并从犯罪人因素和环境因素等方面对犯罪热点内的案件特征结构进行了分析。结 果表明: 北京市内城六区的抢劫案件存在有 “a” “b” “c”三个主要的空间热点, 并且热点内的大部分案件均具有显著 的时空临近重复效应; 其中热点 “a”位于双井、劲松一带, 热点 “c”位于南四环大红门桥一带, 且 2 个热点内案件的 犯罪人特征在一致性程度上高于环境类特征, 表明热点的形成源于犯罪人在热点区域内重复作案的可能性较大; 而热点 “b”位于东南三环的分钟寺地区, 热点内案件的环境类特征在一致性程度上高于犯罪人特征, 表明该热点的 形成为不同犯罪人在热点区域内集中作案的可能性较高。研究对警务部门开展针对性的犯罪打击和防控有一定 的支撑作用。
\end{abstract}

关 键 词: 时空临近重复效应; 犯罪热点; Simpson指数; 案件链; 北京

犯罪活动在空间上的分布并不是均匀的,而是 呈现为在某些区域聚集的形态, 这些犯罪活动高度 聚集的区域一般被称为 “犯罪热点” ${ }^{[1]}$ 。犯罪热点是 犯罪活动在空间上的一种典型分布特征, 通过对犯 罪热点现象进行研究, 可以得到犯罪活动的一般性 规律, 进而从城市规划和人文地理的角度进一步挖 掘犯罪空间分布的原因, 有助于警务部门在打击和 预防犯罪过程中作出科学的决策 ${ }^{[2]}$ 。近些年来, 国 内外研究人员在犯罪的空间聚集和热点现象方面 开展了大量实证分析, 对犯罪热点的分布特征及其 影响因素进行了深人研究。例如, Messner 等 ${ }^{[3]}$ 观察 到美国圣路易斯地区的犯罪热点主要分布在研究

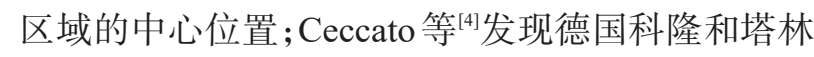
市的抢劫案件主要集中在市中心地区, 并多分布于 车站、酒吧等地; 冯健等 ${ }^{[5]}$ 通过研究侵财类犯罪的空 间分布,发现北京市城八区内的犯罪活动总体上集 中于商业繁华地区、对外交通运输区等强度带, 呈 多中心分布的特征; 朱艳丽等 ${ }^{[6]}$ 研究了美国费城市 抢劫案件的空间热点分布, 发现犯罪热点主要分布 在城区的北部和东北部地区。此外,一些学者还发 现犯罪的空间集中性现象主要受社会、经济、环境 等因素的显著影响, 如 Neumayer ${ }^{[7]}$ 认为居民收人的 不平衡会影响暴力侵财犯罪的空间分布; Wing 等 ${ }^{[8]}$ 认为结合社会经济、人口、环境等数据进行分析有

收稿日期: 2019-10-12; 修订日期: 2020-02-25。

基金项目: 北京市自然科学基金项目(9192022); 社会安全风险感知与防控大数据应用国家工程实验室主任基金项目; 中国人 民公安大学 2019 年拔尖人才培养专项资助硕士研究生科研创新项目(2019ssky002)。 [Foundation: National Natural Science Foundation of Beijing, No. 9192022; National Engineering Laboratory Director Fund for Social Security Risk Perception and Prevention and Control of Large Data Applications; Top Talents Training Specialized Subsidy for Scientific Research and Innovation Projects of Master's Graduates of People's Public Security of China in 2019, No. 2019ssky002. ] 第一作者简介: 郭雅琦(1996-), 女, 河南郑州人, 硕士生, 主要从事犯罪地理、文本挖掘方面的研究。E-mail: hnpyagyq@163.com *通信作者简介: 陈鹏(1981-), 男,辽宁营口人,博士, 副教授,主要从事犯罪地理研究、犯罪情报分析等工作。

E-mail: chenpeng@ppsuc.edu.cn

引用格式: 郭雅琦, 陈鹏. 基于时空临近重复效应的犯罪热点特征及成因分析: 以北京市抢劫案件为例 [J]. 地理科学进展, 2020, 39(5): 804814. [Guo Yaqi, Chen Peng. Formation and feature analyses of crime hotspots using near repeat principle: A case study of robbery in Beijing. Progress in Geography, 2020, 39(5): 804-814. ] DOI: 10.18306/dlkxjz.2020.05.009 
助于确定犯罪空间分布的潜在影响因素; 毛媛媛 等 ${ }^{[9]}$ 对上海市刑事犯罪空间分布与环境因素的关系 分析表明,用地性质、零售业和餐饮业单位的密集 度等均与犯罪的空间分布存在相关关系; 柳林等 ${ }^{[10]}$ 发现 ZG 市计骗犯罪的空间分布与当地的土地利用 混合度、受教育水平等建成环境和社会环境因素存 在高度关联性, 等等。从关于犯罪热点的已有研究 工作来看, 当前主要集中于从实证角度分析和发现 犯罪热点的分布模式,并利用城市规划、地理学等 理论,在基于行政区划的空间尺度下(如区、街道等) 分析犯罪热点的社会、人文、环境、经济性影响要 素, 但对犯罪热点内部空间尺度下犯罪活动的特征 构成及其形成过程还缺少较为深人的分析和挖掘。

在犯罪学研究中, 时空临近重复效应是犯罪活 动一个十分重要的时空特征。所谓犯罪的时空临 近重复效应是指在某个地点发生案件之后, 一定时 间段内距离该地附近一定空间范围内的地点以较 大概率再次发生同类案件的现象 ${ }^{[1]}$ 。近年来, 人们 通过实证研究发现不同类型的案件均存在着一定 的时空临近重复效应,例如, Ratcliffe 等 ${ }^{[12]}$ 证明美国 费城的枪击案件存在有显著的时空临近重复现象, Block 等 ${ }^{[13]}$ 发现美国纽瓦克市的机动车盗窃案件存 在着显著的时空临近重复特征模式。而在描述犯 罪活动的时空临近重复效应机理方面, 人们从犯罪 人员及犯罪区域特征等角度进行了相应的解释。 其中在犯罪人员特征方面, 研究人员发现具有时空 临近重复性特征的案件中有相当大比例的案件为 同一罪犯所为 ${ }^{[14-15]}$, 因此人们认为犯罪人的系列犯 罪现象是形成犯罪时空临近重复效应的原因 ${ }^{[11,16]}$ 。 而在犯罪区域特征方面, 有学者则认为一些区域的 功能和结构本身对犯罪人形成了较大的吸引力, 导 致不同类型的犯罪人在热点区域内集中作案, 从而 形成局部区域短时间内案件高发 ${ }^{[17-21]}$ 。因此, 时空 临近重复效应也被人们认为是犯罪热点形成的原 因之一。近年来, 一些研究人员开始尝试通过分析 犯罪活动的时空临近重复效应, 从犯罪热点的局部 空间尺度来解释犯罪热点的形成, 如 Haberman 等 ${ }^{[22]}$ 和Chainey 等 ${ }^{[23]}$ 发现在一些犯罪热点中部分案件具 有十分显著的时空临近重复特征; 吴玲等 ${ }^{[24]}$ 通过分 析武汉市人室盗窃案件的报警数据, 发现武汉市的 人室盗窃犯罪在一定空间和时间区间内具有时空 临近重复效应, 且连续发生的盗窃案件由同一犯罪 人实施的可能性较高; 徐冲等 ${ }^{[25]}$ 则通过分析 DP 半
岛的抢劫案件发现, 具有时空临近重复效应的案件 在特定空间环境下存在着明显的集聚现象,等等。 但从目前已有的工作进展来看, 基于时空临近重复 效应的犯罪热点研究仍主要集中于对热点内犯罪 活动的时空临近重复效应的验证和识别, 尚缺少基 于犯罪人或环境因素的犯罪热点特征结构与成因 分析, 对犯罪热点的解释性有所不足。

随着智慧警务战略的提出和实施, 对犯罪进行 精准化打击和防控已经成为公安工作发展的重要 方向, 因此对犯罪的分析越来越趋向于精细化。为 了进一步从犯罪案件的特征因素层面了解犯罪热 点的形成过程与原因, 本文拟从犯罪活动的时空临 近重复效应角度研究犯罪热点内部的案件构成, 并 结合案件的犯罪人及环境因素分析犯罪热点的特 征及成因, 为犯罪热点的打击和防控提供相应的理 论支撑, 并丰富犯罪地理学的研究内容。

\section{1 研究框架、研究区域与数据}

\section{1 研究框架}

本文研究框架如图 1 所示。由于本文旨在通过 犯罪的时空临近重复效应分析来研究犯罪热点的 特征和成因, 因此在具体实施过程上分为 2 个步 骤。一方面利用核密度估计来获取案件宏观空间 态势分布以识别显著性的案件热点区域; 另一方面 对案件进行时空临近重复效应分析, 以得到案件整 体的时空临近重复特征模式, 从而根据时空临近重 复效应的参数确定时空区间,在此基础上构建模型 筛选出所有符合时空临近重复效应的案件链。随 后, 提取出犯罪热点区域中满足时空临近重复效应 的案件链,利用案件关联的犯罪人及环境等特征因 素分析犯罪热点的特征模式,并结合时空临近重复 效应解释理论分析犯罪热点的形成原因, 从而给出 相应的犯罪防控对策。

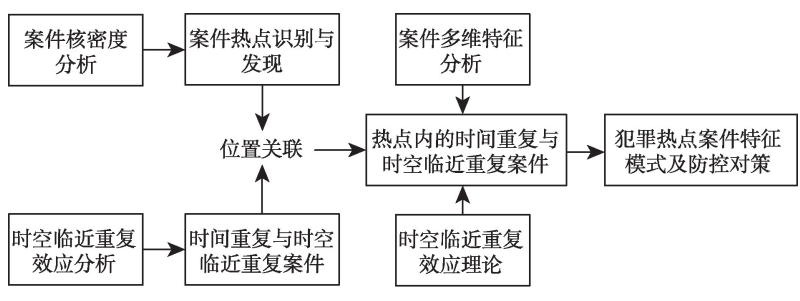

图 1 本文研究框架

Fig.1 Research framework 


\section{2 研究区域}

本文选取北京市为研究区域。北京为中国典 型的超大型城市, 人口密度大, 治安案件多, 具有一 定的代表性。具体研究范围为北京市内城六区(如 图 2 所示),包括东城区、西城区、朝阳区、海淀区、丰 台区和石景山区。北京市内城六区总面积为 $1368.32 \mathrm{~km}^{2}$, 约占全市面积的 $8.3 \%$ 。截至 2014 年, 城六区的常住人口为 1276.3 万人, 约占全市常住人 口的 $59.3 \%$, 常住人口密度约为 9327 人 $/ \mathrm{km}^{2}$, 约为其 他城区常住人口平均密度的 16 倍。由于人口数量 多、密度大且经济发展水平相对较高, 虽然城六区 的治安管理机制较为完善, 但其案发数量仍占有较 高的比重。

\section{3 研究数据}

本文研究数据为北京市 110 接处警系统中 2012-2014年的抢劫类案件数据。抢劫是指犯罪 人使用暴力、胁迫或其他方法, 强行将所有人、保管 人、看护人或持有人的公私财物抢走的行为。选择 该类案件的主要原因在于抢劫案件涉及了危及受 害人生命的暴力行为, 被认为是城市地区人们恐惧 的主要来源 ${ }^{[26]}$ 。由获取的案件数据可知, 北京市城 六区 2012-2014 年发生的抢劫案件数量约占全市 发案总量的 $66.1 \%$ 。本文所选研究区域内的抢劫案 件数据共 2372 条,案件信息字段包括“案件编号” “发案时间” “经度” “纬度”“作案手段” “发案处所” “发案部位”及简要案情描述等。最后, 对数据预处 理及去重后得到的有效案件数据为 1323 条。

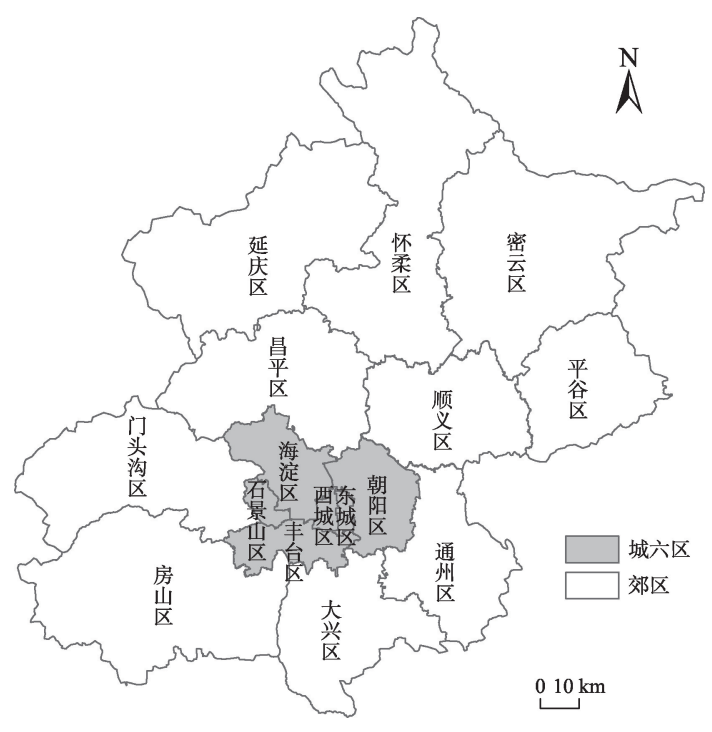

图 2 研究区域示意图

Fig.2 The location of the study area in Beijing

\section{2 研究方法}

根据研究的整体框架流程, 本文分别采用核密 度估计、时空临近重复计算、时间重复及时空临近 重复案件链定义模型及 Simpson 指数等分析方法开 展研究。

\section{1 核密度估计}

核密度是一种非参数估计的空间统计方法。 其原理为: 若视野中出现一个点, 则认为这个点的 概率密度较大, 距离该点越近的点, 则被认为概率 密度也会比较大。其原理与具体实施步骤如下:

（1）假设每一个案件属于独立同分布, 则每个 案件数据点服从概率密度函数, 根据核密度估计原 理,犯罪活动在空间的分布可以表示为区域内所有 犯罪活动邻域内的核密度函数之和 ${ }^{[27]}$ 。设某点 $x_{i}$ 的 核密度函数 $f(s)$ 为:

$$
f(s)=\frac{1}{n} \sum_{i=1}^{n} K\left(\frac{x_{i}-x_{k}}{h}\right)
$$

式中: $x_{k}$ 表示 $x_{i}$ 邻域内的点; $x_{i}-x_{k}$ 表示两者之间的距 离; $K\left(\frac{x_{i}-x_{k}}{h}\right)$ 为核函数; $h$ 为距离衰减阈值, 即带宽。

(2) 核函数的选择。一般而言, 只要平滑峰值 函数满足曲线下方面积为 1 , 即可作为核函数 ${ }^{[28]}$ 。 为保证后期波峰波谷叠加的方便性,本文选择常用 的高斯分布函数作为核函数:

$$
K(x)=\frac{1}{\sqrt{2 \pi}} \exp \left(-\frac{1}{2} x^{2}\right)
$$

(3) 带宽的选择。带宽的选择并无确切的定 义,一般而言通过实际操作, 根据需求选择合适的 带宽。选择较小的带宽能体现空间分布的局部特 征,选择较大的带宽能使热点区域在全局体现得更 明显 ${ }^{[29]}$, 因此, 应根据实际需求选择合适的带宽。

\section{2 时空临近重复特征计算}

时空临近重复特征计算是一种基于蒙特卡洛 (Monte Carlo)模拟方法分析散点数据时空临近特征 的分析方法 ${ }^{[25]}$ 。该方法的分析步骤如下:

(1) 对 $N$ 个事件 $S_{t, v}$, 分别计算每个事件 $S_{t, v}^{i}$ 至其 他 $N-1$ 个事件的时间距离 $\delta \mathrm{t}_{i j}$ 和空间距离 $\delta 1_{i j}(j=1$, $2, \cdots, N, j \neq i)$;

(2) 设定不同的时间与空间临界值 $\Delta T_{k}$ 与 $\Delta L_{k}(k=$ $0,1, \cdots, m)$, 根据事件 $S_{t, v}^{i}$ 与事件 $S_{t, v}^{i}$ 的时间距离 $\delta \mathrm{t}_{i j}$ 与 空间距离 $\delta 1_{i j}$ 计算位于不同时空临界值范围内的事 件对 $\Gamma_{i j}(i=0,1, \cdots N ; j=0,1, \cdots, N, j \neq i)$ 的数量, 最终 
形成一个 $m \times m$ 的矩阵 $\boldsymbol{\varphi}$;

(3) 采用蒙特卡洛仿真方法进行检验, 即先假 定事件之间的时间距离与空间距离呈相互独立状 态,在保持事件空间信息不变的基础上,随机重排 时间信息并按步骤(1)和(2)重新统计矩阵 $\boldsymbol{\varphi}$ 中各要 素的数量, 然后计算结果的置信度 $p$ :

$$
p=1-\frac{n_{e}}{n_{s}+1}
$$

式中: $n_{e}$ 为对应时间临界值 $\Delta T_{k}$ 与空间临界值 $\Delta L_{k}$ 范 围内实际事件对数量大于模拟事件对数量的次数, $n_{s}$ 为蒙特卡洛模拟的次数。模拟次数越多, 则结果 的置信度越高。

\section{3 时间重复与时空临近重复案件链定义模型}

本文借鉴徐冲等 ${ }^{[25}$ 提出的犯罪时空临近重复 的发生流程定义时间重复与时空临近重复案件点 对。时间重复案件点对是指一定时间间隔内在同 一地点先后发生的案件点对, 时空临近重复案件点 对是指在一定时间间隔和空间间隔内先后发生的 案件点对。其中, 以“前案件点” “后案件点” 的概念 解释案件点对的构成, “前案件点”先于并影响“后 案件点”的发生(图 3)。

符合时间重复和时空临近重复关系的一系列 “前案件点”和“后案件点”即构成案件链。根据时 空临近重复计算得出的时空间隔参数, 即可确定时 间重复与时空临近重复案件链的定义规则。定义 时间重复案件链为, “后案件点”与“前案件点” 发生 在同一地点, 且两者时间间隔小于既定的时间间隔 参数。定义时空临近重复案件链为, “后案件点”与 “前案件点”的时空间隔小于既定的时空间隔参 数。此处将各案件点的经纬度坐标转换为高斯坐 标, 则对于“前案件点” $\left(i\left(x_{i}, y_{i}\right), t_{i}\right)$ 和“后案件点” $\left(j\left(x_{j}\right.\right.$, $\left.\left.y_{j}\right), t_{j}\right)$, 其空间间隔 $D$ 为:

$$
D=\left|x_{i}-x_{j}\right|+\left|y_{i}-y_{j}\right|
$$

时间间隔 $T$ 为:

$$
T=t_{j}-t_{i}
$$

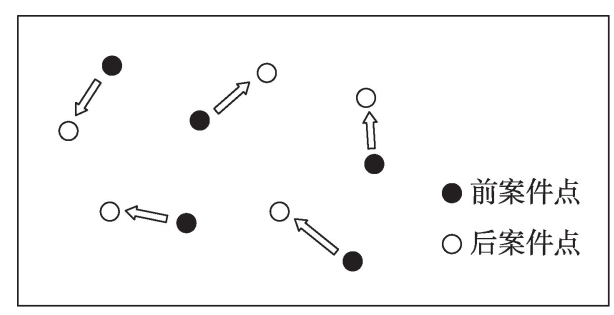

图 3 案件点对模型示意图

Fig.3 Schematic diagram of case pairs

\subsection{Simpson指数}

Simpson 指数多用于生物学领域, 表示同一群 落里随机抽取的 2 个生物样本可能为同一物种的概 率, 旨在表明生物的多样性、均匀性。本文利用 Simpson 指数研究同一案件链中案件特征的异质 性。对于某一案件特征, 若一个案件链中 $C$ 个案件 的该特征中有 $n$ 类不同特征子类, 且有 $C_{j}$ 个案件的 该特征属于第 $j$ 类特征子类, 则 Simpson 指数 $(S)$ 计 算公式为:

$$
S=\frac{\sum_{j=1}^{n} C_{j} \times\left(C_{j}-1\right)}{C \times(C-1)}
$$

若 Simpson 指数越小, 同一案件链中案件特征 的一致性程度越低, 反之Simpson 指数越大, 则同一 案件链中案件特征的一致性程度越高。

\section{3 结果分析}

\section{1 案件的犯罪热点识别}

利用 ArcGIS 10.2 软件,采用核密度估计的方 法对北京市内城六区的抢劫案件进行空间热点识 别, 得到案件的热点分布如图 4 所示。从图 4 中可 以看出, 北京市内城六区的抢劫案件呈现出不均衡 的空间分布态势, 形成了 3 个比较重要的犯罪热点 区域,本文分别以“a”“b”“c”进行命名。3个热点区 域的基本情况分别如下:

热点“a” : 位于北京市东三环朝阳区国贸南的 双井、劲松一带, 热点内共发生案件 35 起。该区域 的北京市常住居民较多, 内有 20 世纪 80 年代建成 的第一批成建制小区, 其住房建筑、环境设施等大 多较为老化, 且基础设施不够健全, 故该区域内环 境对于犯罪人而言有较多的犯罪机会。另一方面, 受 $\mathrm{CBD}$ 核心地带的影响,近年来该区域的经济也得 到快速发展, 各式高档小区、娱乐综合型商场等建 筑纷纷落地建成, 在此地犯罪可得到相对较高的收 益。此外,该地交通通达, 已建成及在建的多条地 铁线都经过此地, 该区域的通达性较高, 犯罪风险 较小, 很容易吸引犯罪人员进行作案 ${ }^{[30]}$ 。

热点 “b”: 位于北京市京津塘高速与南三环黄 金夹角位置的分钟寺一带, 热点内共发生案件 32 起。该区域由于长期以来规划与实际建设的不同 步, 其功能定位仍为城中村, 与其他快速发展的同 期主城区仍有一定差距。截至2014年,该区域内房 价租金较低, 各类公寓、小饭店遍布, 道路狭窄、人 


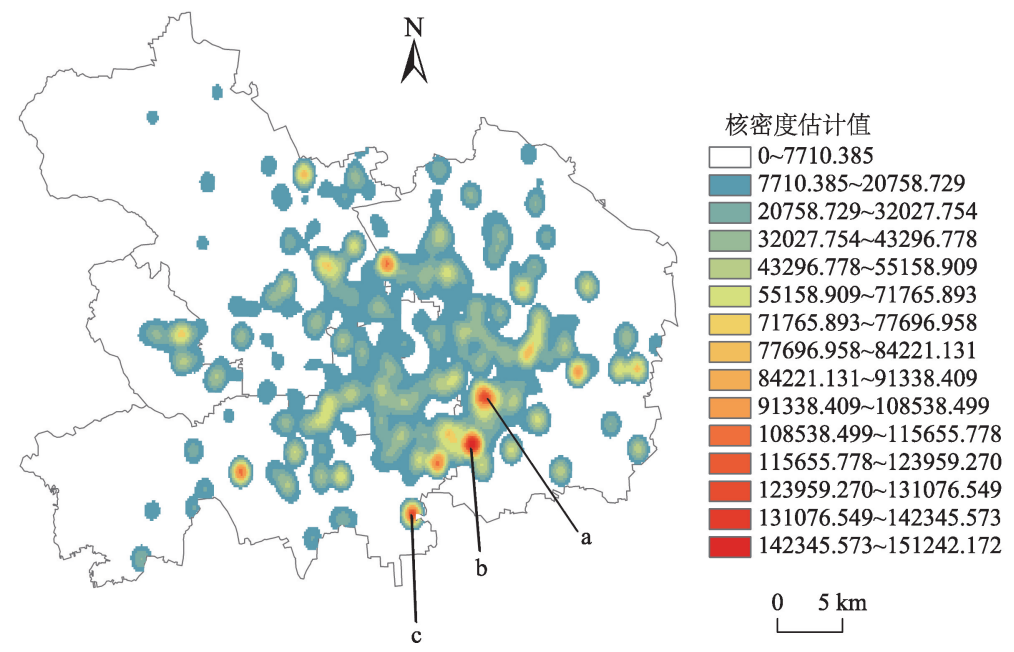

图 4 基于核密度估计的案件热点分布

Fig.4 Distribution of crime hotspots based on kernel density estimation

员众多, 居民生活质量不高, 整体社区环境仍亟待 改造。区域内居住人员也较为繁杂, 文化水平普遍 较低,拥有各类身份职业的人聚集于此, 是流动人 员的集散地。

热点“c”: 位于北京市南四环的大红门桥南苑 路一带, 热点内共发生案件 27 起。该区域北与中心 城区相接, 南为南苑机场。该区域内有著名的大红 门服装商贸区, 是北京市重要的商流、物流、人流和 信息流的集散地,形成了以批发流通为主、带动产 销的商品流通市场体系。相对方便的交通为犯罪 人提供了较高的可达性和便利性, 同时该地人口流 动性大, 社会控制弱, 极易成为犯罪分子的目标 ${ }^{\left[{ }^{[1]}\right.}$ 。

\section{2 案件的时间重复及时空临近重复特征分析}

对各案件经纬度进行高斯坐标转换, 并将各案 件的发案时间单位对应精确到天, 采用临近重复计
算器进行时间重复及时空临近重复特征分析。其 中, 空间带宽设置为 $1000 \mathrm{~m}$, 时间带宽设置为 $7 \mathrm{~d}$, 空间距离采用曼哈顿距离测量法。计算得到的抢 劫案件时间重复及时空临近重复的实际案件点对 数量如表 1 所示, 实际案件分布的临近重复计算值 与模拟期望值之比如表 2 所示。

临近重复计算值与蒙特卡洛模拟得到的平均 期望值之比可以表示特定空间和时间带宽下再次 发生同类案件的犯罪风险。根据表 2 中结果, 北京 市内城六区的抢劫案件呈现出了显著的时间重复 和时空临近重复效应, 其中, 某地点发生抢劫案件 后, 在接下来 $7 \mathrm{~d}$ 之内同一地点再次发生抢劫案件 的风险高达 5.11 , 与完全随机发生案件的风险相比 ${ }^{(1)}$, 犯罪风险增加了 $411 \%$, 且在 $P=0.001$ 的水平上显 著, 表现出了明显的时间重复性发案特征。而在某

表 1 时间重复及时空临近重复案件点对数量

Tab.1 The number of case pairs

\begin{tabular}{lrrrrrrrr}
\hline \multirow{2}{*}{ 空间间隔 $/ \mathrm{m}$} & \multicolumn{7}{c}{ 时间间隔/d } \\
\cline { 2 - 8 } & $(0,7]$ & $(8,14]$ & $(15,21]$ & $(22,28]$ & $(29,35]$ & $(36,42]$ & $(43,49]$ & $>49$ \\
\hline 同一地点 & 182 & 46 & 43 & 33 & 42 & 32 & 36 & 2351 \\
$(1,1000]$ & 59 & 23 & 32 & 11 & 19 & 19 & 33 & 989 \\
$(1001,2000]$ & 42 & 39 & 42 & 45 & 33 & 53 & 59 & 2691 \\
$(2001,3000]$ & 70 & 89 & 50 & 59 & 58 & 62 & 59 & 3918 \\
$(3001,4000]$ & 106 & 81 & 63 & 73 & 98 & 79 & 69 & 5349 \\
$(4001,5000]$ & 98 & 121 & 87 & 95 & 104 & 90 & 78 & 6260 \\
$>5000$ & 10723 & 11182 & 11291 & 11114 & 11011 & 11184 & 10852 & 772976 \\
\hline
\end{tabular}

(1) 在不考虑空间异质性影响犯罪发生的假设条件下,空间上任一地点均存在随机发生案件的可能,若时空临近重复计算结果大于 1.20 且在 0.05 水平上统计显著,则认为具有明显的时空临近重复效应。 
表 2 实际案件分布的临近重复计算值与模拟平均期望值之比及其显著性水平

Tab.2 Observed over mean expected frequencies and significance levels

\begin{tabular}{|c|c|c|c|c|c|c|c|c|}
\hline \multirow{2}{*}{ 空间间隔/m } & \multicolumn{8}{|c|}{ 时间间隔/d } \\
\hline & $(0,7]$ & $(8,14]$ & $(15,21]$ & $(22,28]$ & $(29,35]$ & $(36,42]$ & $(43,49]$ & $>49$ \\
\hline 同一地点 & $5.11^{* *}$ & 1.25 & 1.17 & 0.92 & 1.17 & 0.87 & 1.02 & 0.94 \\
\hline$(1,1000]$ & $3.87^{* * *}$ & $1.47^{*}$ & $2.00^{* * *}$ & 0.70 & 1.23 & 1.21 & $2.19^{* *}$ & 0.92 \\
\hline$(1001,2000]$ & 1.09 & 0.98 & 1.06 & 1.14 & 0.84 & $1.33^{*}$ & $1.54^{*}$ & 0.99 \\
\hline$(2001,3000]$ & $1.24^{*}$ & $1.54^{*}$ & 0.86 & 1.03 & 1.02 & 1.08 & 1.05 & 0.99 \\
\hline$(3001,4000]$ & $1.40^{* *}$ & 1.03 & 0.80 & 0.94 & $1.27^{*}$ & 1.01 & 0.91 & 0.99 \\
\hline$(4001,5000]$ & 1.10 & $1.32^{* *}$ & 0.95 & 1.04 & 1.15 & 0.99 & 0.88 & 0.99 \\
\hline$>5000$ & 0.98 & 0.99 & 1.00 & 1.00 & 1.00 & 1.00 & 1.00 & $1.00^{\circ}$ \\
\hline
\end{tabular}

注: ***分别表示通过显著性水平为 $0.05 、 0.001$ 的检验。

案发地点以外至周边 $1000 \mathrm{~m}$ 范围内、案发后 3 个星 期内再次发生抢劫案件的风险分别为 $3.87 、 1.47$ 、 2.00 , 其数值均高于 1.20 , 且显著性水平 $P$ 均小于 0.05 , 表现出了明显的时空临近重复性发案特征。 此外, 虽然距离抢劫案件发生地点 2000 3000 m 之 间、案发后 2 周内再次发生案件的风险达到了 1.24 和 1.54, 但在距案发地点 1000 2000 m 区域之间再 次发生案件的风险较低,形成了一个风险缓冲区。 因此, 根据表中结果确定时间重复性发案条件为: 某地点发生抢劫案件之后的 $7 \mathrm{~d}$ 内同一地点再次发 生同类案件; 时空临近重复性发案条件为: 距离发 生上一起抢劫案件的地点至周边 $1000 \mathrm{~m}$ 区域范围 内、接下来 $21 \mathrm{~d}$ 内再次发生同类案件。根据结果, 定义时间重复性案件链“后案件点”与“前案件点” 的时间间隔参数为 $7 \mathrm{~d}$, 定义时空临近重复性案件 链 “后案件点”与 “前案件点” 的时间间隔参数为 $21 \mathrm{~d}$ 、空间间隔参数为 $1000 \mathrm{~m}$ 。

\section{3 案件链的构建和提取}

\subsection{1 时间重复、时空临近重复案件链}

根据 2.3 中关于时间重复和时空临近重复案件 链的定义方法, 利用表 2 中得到的时空间隔参数提 取所有满足条件的 2 种案件链。最终得到案件链共 188 条, 其中时间重复案件链 78 条, 时空临近重复
案件链 110 条, 具体结果如表 3 所示。

从表 3 可以看出, 链长为 2 的案件链数量最多, 共有 114 条, 达到总数的 $60.6 \%$ 。随着链中案件数 量的增多, 案件链的数量逐步递减, 并在链中案件 数量为 $7 、 8 、 9 、 10$ 时出现断层。而当链中案件数量 为 11 时,其案件链数仅为 1 。由此可以认为: 时间 重复和时空临近重复案件链的长度大多为 2 或 3 , 这意味着北京市内城六区抢劫案件的时间重复及 时空临近重复性发案现象虽较为明显,但“前案件 点” 的影响并不广泛, 这也基本符合抢劫案件的特 征。不同于人室盗窃犯罪受害地点具有固定性, 抢 劫案件的受害人与受害地点有着随机、偶然且多变 的特点 ${ }^{[2]}$, 因此抢劫类案件的链长度往往较为有限。

\subsection{2 热点内案件的时空临近重复效应分析}

将案件链与热点区域进行位置关联, 得到热点 “a” “b” “c”中符合时间重复或时空临近重复效应的 案件共 72 起, 其中时间重复性案件 24 起, 时空临近 重复性案件 48 起,共形成案件链 76 条, 其中时间重 复案件链 8 条, 时空临近重复案件链 68 条。可以看 出, 虽然犯罪热点中具有时间重复效应与具有时空 临近重复效应的案件数量相差不大, 但时空临近重 复案件链的数量远多于时间重复案件链, 即时空临 近重复性案件较时间重复性案件的“前案件点”对

表 3 时间重复、时空临近重复案件链数量与占比情况分布

Tab.3 The number and proportion of case chains

\begin{tabular}{|c|c|c|c|c|c|c|c|c|c|c|}
\hline \multirow{2}{*}{ 统计项 } & \multicolumn{10}{|c|}{ 链中案件数量 } \\
\hline & 2 & 3 & 4 & 5 & 6 & 7 & 8 & 9 & 10 & 11 \\
\hline 时间重复案件链数量/条 & 62 & 8 & 6 & 1 & 0 & 0 & 0 & 0 & 0 & 1 \\
\hline 时空临近重复案件链数量/条 & 52 & 14 & 12 & 16 & 16 & 0 & 0 & 0 & 0 & 0 \\
\hline 案件链数量总计/条 & 114 & 22 & 18 & 17 & 16 & 0 & 0 & 0 & 0 & 1 \\
\hline 在所有案件链中占比/\% & 60.6 & 11.7 & 9.6 & 9.0 & 8.5 & 0 & 0 & 0 & 0 & 0.5 \\
\hline
\end{tabular}


“后案件点”的影响程度更大、范围更广。各犯罪热 点内的时间重复性案件及其案件链、时空临近重复 性案件及其案件链的数量统计结果如表4所示。

由上文分析可知, 热点 “a”、 “b”、“c”中的案件 数量分别为 $35 、 32 、 27$ 起。设热点 $i$ 的案件数量为 $N$, 其中符合时间重复或时空临近重复效应的案件 数量为 $n_{i}$, 则

$$
p_{i}=\frac{n_{i}}{N} \times 100 \%
$$

式中: $p_{i}$ 为热点 $i$ 内部的时空临近重复效应程度。 得 $p_{1}=74.29 \%, p_{2}=100.00 \%, p_{3}=51.85 \%$ 。可见, 在北 京市内城六区形成的犯罪热点中大部分案件都具 有时空临近重复效应, 其中热点“b”内的案件全部 符合时空临近重复效应, 这与 Haberman 等 ${ }^{[22]}$ 提出的 “犯罪的时空临近重复效应导致多个事件构成的案 件链有可能促成犯罪热点的形成”这一假设相一 致。因此可以认为, 犯罪的时空临近重复效应是北 京市内城六区 2012-2014 年间发生的抢劫案件犯 罪热点形成的主要原因。

\section{4 犯罪热点内案件的特征分析}

根据犯罪活动时空临近重复效应的理论分析, 一般认为犯罪人因素和环境因素是导致犯罪时空 临近重复效应的 2 个主要原因, 其中犯罪人因素主 要表现为犯罪人曾经成功实施的犯罪经历会对其 自身形成心理暗示效应, 促使其再次回到原案发 地点实施犯罪, 而环境因素则表现为区域自身存 在多个吸引犯罪的因素导致犯罪人到区域内集中

表 4 犯罪热点内案件、案件链数量对应

Tab.4 The number of cases and case chains located within crime hotspots

\begin{tabular}{cccc}
\hline \multicolumn{1}{c}{ 案件/案件链 } & 热点“a” & 热点“b” & 热点“c” \\
\hline 时间重复案件 & 9 & 9 & 6 \\
时间重复案件链 & 4 & 2 & 2 \\
时空临近重复案件 & 17 & 23 & 8 \\
时空临近重复案件链 & 14 & 50 & 4 \\
\hline
\end{tabular}

作案 ${ }^{[2-34]}$ 。对此, 根据案件信息, 分别从案件的犯罪 人因素和环境因素 2 个方面对犯罪热点内的案件特 征进行分析。北京市抢劫案件的报警信息中包含 有“发案时间”“作案手段”“发案处所”“发案部位” “被抢物品类型”等特征, 其中“发案时间” “作案手 段”能够反映出犯罪人的作案习惯以及在犯罪过程 中的偏好性 ${ }^{[35]}$, 若热点内案件的发案时间和作案手 段趋于一致则反映出热点内案件很大可能为犯罪 人系列作案, 因此将其归为 “犯罪人因素”。而“发 案处所” “发案部位” “被抢物品类型”等与区域环境 结构和受害人更为相关, 若这几类特征具有较高的 一致性则表明很可能是区域内存在有高价值犯罪 目标或高集中犯罪机会导致了案件高发,因此将其 归为 “环境因素”。每一类因素的案件特征类型及 其对应特征子类如表 5 所示。

随后, 利用式(6), 分别对每个犯罪热点中符合 时间重复或时空临近重复效应的案件计算其犯罪 人因素和环境因素的 Simpson指数,计算结果如表 6 所示。从分析结果中可见, 对热点“a”, “发案时 间”和“作案手段”等表征犯罪人因素特征的平均 Simpson 指数分别为 0.648 和 0.444 ,均明显高于 “发 案处所 $(0.074)$ ”“发案部位 $(0.019)$ ”和“被抢物品类 型(0.148)”等表征环境因素特征的平均 Simpson 指 数, 即热点 “a” 的犯罪人因素表现更为显著; 对热点 “b”, 热点内案件各项特征的 Simpson 指数均偏低, 但相对比较来看, 在表征环境因素的几项特征中, 除“发案处所(仅为 0.085 )”外, “发案部位( 0.347 )”和 “被抢物品类型 $(0.447)$ ”的平均 Simpson 指数均高于 “发案时间 $(0.287)$ ”和“作案手段 $(0.146)$ ”, 即热点 “b”的环境因素表现得更为明显; 而对热点 “c”, 其 表征犯罪人因素特征的平均 Simpson 指数均高于环 境因素的几项特征, 表明热点 “c”中案件的犯罪人 因素表现得更为明显, 其案件特征结构与热点 “a” 相似。

表 5 犯罪人及环境因素案件特征分类

Tab.5 Categories of case characteristics of criminal and environmental factors

\begin{tabular}{lll}
\hline 案件因素类型 & 案件特征类型 & \\
\hline 犯罪人因素 & 发案时间 & 凌晨/早晨/上午/中午/下午/晚上 \\
& 作案手段 & 踢打/言语胁迫/扼颈/持锐器/仗势胁迫/露械胁迫/强拿硬要/持钝器/搜身 \\
& 发案处所 & 宾馆/高层楼房/商业区/居民小区/网吧/普通楼房/楼群/贸易市场/一般公路/乡村公路/广场/结合部/出租 \\
& & 屋/树林/商店/建筑工地/平房/街巷 \\
& 发案部位 & 客房/电梯间/路旁/楼道/胡同/卧室/河边/地下室/街心公园/门前/出租房屋/工地料场/楼口/路口/车厢 \\
& 被抢物品类型 & 卡/电子产品/钱/包/首饰/日用品/证件/车/钥匙/衣物 \\
\end{tabular}


表 6 热点各项案件特征的 Simpson 指数

Tab.6 Simpson index of case characteristics of crime hotspots

\begin{tabular}{|c|c|c|c|c|c|c|}
\hline \multirow{2}{*}{ 热点编号 } & \multirow{2}{*}{ 统计指标 } & \multicolumn{2}{|c|}{ 犯罪人因素 } & \multicolumn{3}{|c|}{ 环境因素 } \\
\hline & & 发案时间 & 作案手段 & 发案处所 & 发案部位 & 被抢物品类型 \\
\hline \multirow[t]{4}{*}{ “a” } & 最大值 & 1.000 & 1.000 & 1.000 & 0.167 & 1.000 \\
\hline & 最小值 & 0 & 0 & 0 & 0 & 0 \\
\hline & 平均值 & 0.648 & 0.444 & 0.074 & 0.019 & 0.148 \\
\hline & 标准差 & 0.431 & 0.435 & 0.244 & 0.054 & 0.317 \\
\hline \multirow[t]{4}{*}{ "b” } & 最大值 & 1.000 & 0.600 & 0.600 & 1.000 & 1.000 \\
\hline & 最小值 & 0 & 0 & 0 & 0 & 0 \\
\hline & 平均值 & 0.287 & 0.146 & 0.085 & 0.347 & 0.447 \\
\hline & 标准差 & 0.196 & 0.136 & 0.089 & 0.192 & 0.269 \\
\hline \multirow[t]{4}{*}{ "c" } & 最大值 & 1.000 & 1.000 & 0.333 & 0 & 1.000 \\
\hline & 最小值 & 0 & 0 & 0 & 0 & 0 \\
\hline & 平均值 & 0.800 & 0.333 & 0.067 & 0 & 0.200 \\
\hline & 标准差 & 0.443 & 0.365 & 0.139 & 0 & 0.400 \\
\hline
\end{tabular}

\section{4 讨论}

通过对北京市内城六区抢劫案件进行分析, 发 现犯罪热点内的大部分案件存在着显著的时空临 近重复效应,表明时空临近重复效应是犯罪热点形 成的主要原因,该结论与以往研究基本一致。而对 于犯罪热点形成的深层次原因, 通过结合相关理论 对热点内案件的特征结构进行一致性分析, 则能够 进一步挖掘热点形成的机制。首先, 旗帜理论认为 局部环境中形成的犯罪机会相对集中,引诱犯罪人 到区域内频繁作案是产生时空临近重复效应的原 因之一。因此, 区域内有助于形成犯罪机会的环境 特征的一致性越强, 则意味着越有可能发生具有时 空临近重复性效应的犯罪活动 ${ }^{[3]}$, 在热点内案件的 特征结构分布上则表现为环境因素的一致性越高， 也即 Simpson 指数越高。从实际情况来看, 2014年 热点“b”所在区域改造前主要为城中村社区,大部 分建筑为平房结构, 环境较为单一, 且区域内外来 人口比重较大, 安防设施覆盖率较低, 社区非正式 监管能力较差, 特别是 2013 年北京地铁 10 号线二 期开通后该区域内设置了分钟寺站,提升了当地的 交通便利性, 为犯罪人提供了更多犯罪机会, 容易 诱使一些具有犯罪企图的人员到当地集中实施犯 罪。因此,可以认为环境特征所造成的犯罪机会集 中是热点 “ b”形成的主要原因。其次, 从另一个角 度来看, 激励理论则认为犯罪人曾经成功犯罪的经 验对其自身形成了心理暗示效应、诱使犯罪人重新 回到犯罪现场附近再次作案是产生时空临近效应 的另一个原因, 即犯罪人对环境的依赖性较小, 更
多取决于其对环境具有较高认知前提下犯罪行为 的选择,具体表现为反映其犯罪行为习惯的犯罪人 作案手段、作案时间等主体性特征的一致性较高。 因此, 若热点内案件特征分布中犯罪人因素的 Simpson 指数越高, 则表明热点内发生案件的犯罪 主体特征可能会趋向于一致,即为犯罪人在热点区 域内重复作案的可能性较大。从实际区域环境结 构上来看,热点“a”毗邻国贸商圈, 区域内高档小区 众多,安防设施覆盖率较高, 而热点“c”内有大红门 大型服装商城, 平日人流众多,这些均不是抢劫案 件发生的理想条件,但却形成了案件的高发态势, 因此,可以认为一些犯罪人在区域内有针对性地重 复作案是热点“a”和“c”形成的主要原因。

\section{5 结论}

本文以北京市内城六区 2012-2014年的抢劫 案件为例, 从犯罪活动时空临近重复性分析的视角 对北京市内城六区抢劫案件的空间热点特征及其 成因进行了研究。结果表明:犯罪时空临近重复效 应是北京市内城六区的抢劫案件热点形成的主要 原因,但对于不同的犯罪热点,其特征和形成原因 有所不同。通过对热点内的犯罪人因素及环境因 素特征进行 Simpson一致性分析, 发现犯罪人因素 表现较为明显的热点为犯罪人系列作案的可能性 较大, 而环境因素表现较为明显的热点为多个犯罪 人到同一区域集中作案的可能性较大。本文针对 不同犯罪热点的人文地理环境、时空临近重复效应 及案件特征的一致性分析, 进一步揭示了犯罪热点 
的结构特征,有助于警务部门对不同类型的犯罪热 点采取具有针对性的打击和防控措施。例如根据 热点的犯罪人因素、环境类因素的特征分布, 对犯 罪人因素特征更明显的, 可加强对热点内案件的串 并案处理和研判, 及时发现潜在的系列犯罪人, 并 根据案件的作案手段和发案时间等特征做好相应 的巡控措施与防范宣传。而对环境类因素特征更 为明显的, 可以有的放矢, 重点围绕热点区域的高 发案部位采取措施, 如提高安防设施的覆盖率或加 强民警巡逻盘查, 以压缩和减少犯罪人的作案空 间等。

\section{参考文献(References)}

[1] Eck J E, Chainey S P, Cameron J G, et al. Mapping crime: Understanding hot spots $[\mathrm{M}]$. Washington D C, USA: National Institute of Justice, 2005.

[2] 徐嘉祥, 陈鹏, 陈建国. 基于环境犯罪学理论的人室盗窃 时空分布研究: 以北京市主城区案件的分析为例 [J]. 人 文地理, 2018, 33(1): 43-50. [Xu Jiaxiang, Chen Peng, Chen Jianguo. Research on spatial-temporal distributions of burglary based on the environmental criminology: Based on the analysis of crime in Beijing. Human Geography, 2018, 33(1): 43-50. ]

[3] Messner S F, Anselin L, Baller R D, et al. The spatial patterning of county homicide rates: An application of exploratory spatial data analysis [J]. Journal of Quantitative Criminology, 1999, 15(4): 423-450.

[4] Ceccato V N, Oberwittler D. Comparing spatial patterns of robbery: Evidence from a Western and an Eastern European city [J]. Cities, 2008, 25(4): 185-196.

[5] 冯健, 黄琳珊, 董颖, 等. 城市犯罪时空特征与机制: 以北 京城八区财产类犯罪为例 [J]. 地理学报, 2012, 67(12): 1645-1656. [Feng Jian, Huang Linshan, Dong Ying, et al. Research on the spatial-temporal characteristics and mechanism of urban crime: A case study of property crime in Beijing. Acta Geographica Sinica, 2012, 67(12): 16451656. ]

[6] 朱艳丽, 靖常峰, 伏家云, 等. 时空立方体的抢劫案件时 空特征挖掘与分析 [J]. 测绘科学, 2019, 44(9): 132-138, 145. [Zhu Yanli, Jing Changfeng, Fu Jiayun, et al. Analysis of space-time pattern of robbery crime based on spacetime cube. Science of Surveying and Mapping, 2019, 44 (9): 132-138, 145. ]

[7] Neumayer E. Inequality and violent crime: Evidence from data on robbery and violent theft [J]. Journal of Peace Re- search, 2005, 42(1): 101-112.

[8] Wing M G, Tynon J. Crime mapping and spatial analysis in national forests [J]. Journal of Forestry, 2006, 104(6): 293-298.

[9] 毛媛媛,戴慎志. 犯罪空间分布与环境特征: 以上海市为 例 [J]. 城市规划学刊, 2006(3): 85-93. [Mao Yuanyuan, Dai Shenzhi. Research on spatial and environmental characters of crimes: Case study of Shanghai. Urban Planning Forum, 2006(3): 85-93. ]

[10] 柳林, 张春霞, 冯嘉欣, 等. ZG 市计骗犯罪的时空分布 与影响因素 [J]. 地理学报, 2017, 72(2): 315-328. [Liu Lin, Zhang Chunxia, Feng Jiaxin, et al. Analysis of spatial- temporal feature of burglary cases in Changchun city. Acta Geographica Sinica, 2017, 72(2): 315-328. ]

[11] Townsley M, Homel R, Chaseling J. Infectious burglaries. A test of the near repeat hypothesis [J]. British Journal of Criminology, 2003, 43(3): 615-633.

[12] Ratcliffe J H, Rengert G F. Near-repeat patterns in Philadelphia shootings [J]. Security Journal, 2008, 21(1-2): 5876.

[13] Block S, Fujita S. Patterns of near repeat temporary and permanent motor vehicle thefts [J]. Crime Prevention \& Community Safety, 2013, 15(2): 151-167.

[14] Bowers K J, Johnson S D. Who commits near repeats? A test of the boost explanation [J]. Western Criminology Review, 2004, 5(3): 12-24.

[15] Bernasco W. Them again? Same-offender involvement in repeat and near repeat burglaries [J]. European Journal of Criminology, 2008, 5(4): 411-431.

[16] Reiss A. Indicators of crime and criminal justice: Quantitative studies $[\mathrm{M}]$. Washington D C, USA: US Department of Justice, Bureau of Justice Statistics, 1980.

[17] Nelson J F. Multiple victimization in American cities: A statistical analysis of rare events [J]. American Journal of Sociology, 1980, 85(4): 870-891.

[18] Spelman W. Once bitten, then what? Cross-sectional and time-course explanations of repeat victimization [J]. The British Journal of Criminology, 1995, 35(3): 366-383.

[19] Morgan F. Repeat burglary in a Perth suburb: Indicator of short-term or long-term risk? [J]. Crime Prevention Studies, 2000, 12: 83-118.

[20] Sidebottom A. Repeat burglary victimization in Malawi and the influence of housing type and area- level affluence [J]. Security Journal, 2012, 25(3): 265-281.

[21] Chainey S P, da Silva B F A. Examining the extent of repeat and near repeat victimisation of domestic burglaries 
in Belo Horizonte, Brazil [J]. Crime Science, 2016, 5(1): 1-10.

[22] Haberman C P, Ratcliffe J H. The predictive policing challenges of near repeat armed street robberies [J]. Policing: A Journal of Policy and Practice, 2012, 6(2): 151166.

[23] Chainey S P, Curtis-Ham S J, Evans R M, et al. Examining the extent to which repeat and near repeat patterns can prevent crime $[\mathrm{J}]$. Policing: An International Journal, 2018, 41(5): 608-622.

[24] $\mathrm{Wu} \mathrm{L}, \mathrm{Xu} \mathrm{X}, \mathrm{Ye} \mathrm{X}$, et al. Repeat and near-repeat burglaries and offender involvement in a large Chinese city [J]. Cartography and Geographic Information Science, 2015, 42(2): 178-189.

[25] 徐冲, 柳林, 周素红. DP 半岛街头抢劫案件的临近重复 发生模式 [J]. 地理研究, 2015, 34(2): 384-394. [Xu Chong, Liu Lin, Zhou Suhong. Patterns of near- repeat street robbery in DP Peninsula. Geographical Research, 2015, 34(2): 384-394. ]

[26] Tompson L. Street robbery [EB/OL]. JDiBrief Series, 2012 [2019-10-02]. http://www.ucl.ac.uk/jdibrief/crime/ street-robbery.

[27] 陈鹏, 李欣, 胡啸峰, 等. 北京市长安街沿线的扒窃案件 高发区分析及防控对策 [J]. 地理科学进展, 2015, 34 (10): 1250-1258. [Chen Peng, Li Xin, Hu Xiaofeng, et al. Clustering pattern analysis and prevention strategies to pickpocketing offence along the Chang'an Street in Beijing. Progress in Geography, 2015, 34(10): 1250-1258. ]

[28] 李存华, 孙志挥, 陈耿, 等. 核密度估计及其在聚类算法 构造中的应用 [J]. 计算机研究与发展, 2004, 41(10): 1712-1719. [Li Cunhua, Sun Zhihui, Chen Geng, et al. Kernel density estimation and its application to clustering algorithm construction. Journal of Computer Re- search and Development, 2004, 41(10): 1712-1719. ]

[29] 禹文豪, 艾廷华. 核密度估计法支持下的网络空间 POI 点可视化与分析 [J]. 测绘学报, 2015, 44(1): 82-90. [Yu Wenhao, Ai Tinghua. The visualization and analysis of POI features under network space supported by kernel density estimation. Acta Geodaetica et Cartographica Sinica, 2015, 44(1): 82-90. ]

[30] Davies T, Johnson S D. Examining the relationship between road structure and burglary risk via quantitative network analysis [J]. Journal of Quantitative Criminology, 2014, 31(3): 481-507.

[31] Sampson R J, Groves W B. Community structure and crime: Testing social-disorganization theory [J]. American Journal of Sociology, 1989, 94(4): 774-802.

[32] Tseloni A, Pease K. Repeat personal victimization. "Boosts" or "flags"? [J]. British Journal of Criminology, 2003, 43(1): 196-212.

[33] Johnson S D. Repeat burglary victimisation: A tale of two theories [J]. Journal of Experimental Criminology, 2008, 4(3): 215-240.

[34] Wells W, Wu L, Ye X. Patterns of near-repeat gun assaults in Houston $[\mathrm{J}]$. Journal of Research in Crime and Delinquency, 2012, 49(2): 186-212.

[35] 韩宁, 陈巍. 基于聚类分析的串并案研究 [J]. 中国人民 公安大学学报(自然科学版), 2012, 18(1): 53-58. [Han Ning, Chen Wei. Research on bunching and merging cases based on cluster analysis. Journal of People's Public Security University of China (Science and Technology), 2012, 18(1): 53-58. ]

[36] Turchan B, Grubb J A, Pizarro J M, et al. Arson in an urban setting: A multi-event near repeat chain analysis in Flint, Michigan [J]. Security Journal, 2019, 32(3): 179197. 


\title{
Formation and feature analyses of crime hotspots using near repeat principle: A case study of robbery in Beijing
}

\author{
GUO Yaqi, CHEN Peng* \\ (Institute of Information Engineering of Policing and Cyber Security, People's Public Security \\ University of China, Beijing 102600, China)
}

\begin{abstract}
As a significant spatiotemporal characteristic of crimes, repeat and near repeat pattern has received much interest in criminology research. The purpose of this study was to explore the formation process and features of crime hotspots by using near repeat principle. Robbery cases in six districts of Beijing inner city from 2012 to 2014 were used to examine the extent to which repeats and near repeats spatially intersect robbery hotspots. All the case chains within crime hotspots satisfying repeat and near repeat principle were screened out. From this, by dividing the case characteristics into criminal factors and environmental factors, the characteristics of case chains were analyzed to describe the features and formation of hotspots. The results suggest that there were three main crime hotspots in the six districts of Beijing inner city, namely "a", "b", "c", and most of the cases located within the hotspots were repeats and near repeats. The hotspot "a" was located in Shuangjing and Jinsong, and the hotspot "c" was located in Dahongmen Bridge. The characteristics of the criminal factors of these two hotspots were more consistent than that of the environmental factors, which indicates that the formation of the hotspots were more likely to originate from the repeated crimes committed in the area by criminals. The hotspot "b" was located in Fenzhongsi area by the southeastern third ring road. The characteristics of the environmental factors of this hotspot were more consistent than that of the criminal factors, which indicates that the formation of the hotspot was more likely to originate from different criminals committing crimes in this area. The research findings presented in this article can aid decision making on crime prevention and detection in policing.
\end{abstract}

Keywords: near repeat principle; crime hotspot; Simpson index; case chain; Beijing 